\title{
Metal-Free Gas Supply at the Edge of Reionization: Late-Epoch Population III Star Formation
}

\author{
Michele Trenti \\ University of Colorado, Center for Astrophysics and Space Astronomy, 389-UCB, Boulder, \\ CO 80309 USA \\ trenti@colorado.edu \\ and \\ Massimo Stiavelli \\ Space Telescope Science Institute, 3700 San Martin Drive, Baltimore, MD 21218 USA \\ mstiavel@stsci.edu \\ and \\ J. Michael Shull \\ University of Colorado, CASA, Dept. of Astrophysical 6 Planetary Sciences, 389-UCB, \\ Boulder, CO 80309 USA \\ michael.shull@colorado.edu
}

\begin{abstract}
While the average metallicity of the intergalactic medium rises above $Z \gtrsim$ $10^{-3} Z_{\odot}$ by the end of the reionization, pockets of metal-free gas can still exist at later times. We quantify the presence of a long tail in the formation rate of metalfree halos during late stages of reionization (redshift $z \approx 6$ ), which might offer the best window to detect Population III stars. Using cosmological simulations for the growth of dark matter halos, coupled with analytical recipes for the metal enrichment of their interstellar medium, we show that pockets of metal-free gas exist at $z \approx 6$ even under the assumption of high efficiency in metal pollution via winds. A comoving metal-free halo formation rate $d^{2} n / d t d V \gtrsim 10^{-9} \mathrm{Mpc}^{-3} \mathrm{yr}^{-1}$ is expected at $z=6$ for halos with virial temperature $T_{v i r} \approx 10^{4} \mathrm{~K}$ (mass $\sim 10^{8} \mathrm{M}_{\odot}$ ), sufficient to initiate cooling even with strong negative radiative feedback. These halos will appear as absorption systems with a typical hydrogen
\end{abstract}


column density of $\sim 10^{20} \mathrm{~cm}^{-2}$, a sky covering factor $5 \times 10^{-3}$ and a number density of $25 \operatorname{arcmin}^{-2}$ for $5.5 \leq z \leq 6.5$. Under the assumption of a single Population III supernova formed per metal-free halo, we expect an observed supernova rate of $2.6 \times 10^{-3} \mathrm{deg}^{-2} \mathrm{yr}^{-1}$ in the same redshift range. These metalfree stars and their supernovae will be isolated and outside galaxies (at distances $\gtrsim 150 \mathrm{~h}^{-1} \mathrm{kpc}$ ) and thus significantly less biased than the general population of $\sim 10^{8} \mathrm{M}_{\odot}$ halos at $z \approx 6$. Supernova searches for metal-free explosions must thus rely on large area surveys. If metal-free stars produce very luminous supernovae, like SN2006gy, then a multi-epoch survey reaching $m_{\mathrm{AB}}=27$ at $1 \mu \mathrm{m}$ is sufficient for detecting them at $z=6$. While the Large Synoptic Survey Telescope (LSST) will not reach this depth in the $\mathrm{z}$ band, it will be able to detect several tens of Population III supernovae in the $\mathrm{i}$ and $\mathrm{r}$ bands at $z \lesssim 5.5$, when their observed rate is down to $3-8 \times 10^{-4} \mathrm{deg}^{-2} \mathrm{yr}^{-1}$.

Subject headings: galaxies: high-redshift - early universe - intergalactic medium - supernovae: general - cosmology: theory - stars: formation

\section{Introduction}

Metal-free stars, conventionally called Population III stars, represent the first step to exit the dark ages of the universe and start the reionization era. The chemical enrichment of the intergalactic medium (IGM) opens the way to the formation of stars and galaxies like our own Milky Way (see Bromm \& Larson 2004). This first step in the star formation history of the Universe has attracted large theoretical interest, and thanks to a combination of analytic and numerical investigations, we now have a reasonably solid understanding of the properties of Population III stars within the $\Lambda C D M$ paradigm. Population III stars are expected to start forming within dark matter halos of mass $\sim 10^{5} \mathrm{M}_{\odot}$ (minihalos) at $z \approx 60$, about 40 Myr after the Big-Bang (Naoz et al. 2006; Trenti \& Stiavelli 2007), most likely with a top-heavy mass function and just one star per halo (Abel et al. 2002; Bromm \& Larson 2004; O'Shea \& Norman 2007, 2008). Once the epoch of Population III stars begins, their formation rate in minihalos increases exponentially with redshift, reaching a level of $10^{-7} \mathrm{Mpc}^{-3} \mathrm{yr}^{-1}$ at $z \approx 25$. Eventually, by $z \approx 15$, Population III star formation in minihalos is suppressed by the build-up of a strong radiative background in the $\mathrm{H}_{2}$ LymanWerner (LW) bands, which photodissociates the molecular hydrogen responsible for the cooling of the primordial gas. A second mode of metal-free star formation is possible in larger halos $\left(M \gtrsim 2 \times 10^{7} \mathrm{M}_{\odot}\right)$ where self-shielding against LW feedback is more effective and where cooling may also be driven by Ly $\alpha$ lines. However as the redshift decreases, it 
becomes progressively more unlikely that $\sim 10^{7} \mathrm{M}_{\odot}$ halos composed of metal-free gas can form (Furlanetto \& Loeb 2005; Trenti \& Stiavelli 2009). The transition to Population II star formation happens in the metallicity range $Z \sim 10^{-3}-10^{-4} Z_{\odot}$ (Bromm et al. 2001a; Santoro \& Shull 2006; Smith et al. 2009) or even at lower metallicity in the presence of dust (Schneider et al. 2006). Pockets of pristine gas are still likely to exist at $z<6$, thanks to the inhomogeneity of metal enrichment in the IGM (Scannapieco et al. 2003; Schneider et al. 2006; Tornatore et al. 2007; Ricotti et al. 2008).

An unambiguous observational test of the Population III paradigm would represent a significant step toward our understanding of star formation in the Universe. This is still missing, despite many attempts ranging from Galactic surveys for metal-poor stars (Beers \& Christlieb 2005; Frebel et al. 2007; Cohen et al. 2008) to surveys aimed at identifying their spectral signatures in galaxies at very high redshift (Nagao et al. 2005, 2008). In fact, Population III stars are expected to be extremely hot and thus strong emitters in the He II lines (Tumlinson \& Shull 2000; Oh et al. 2001). Some indirect evidence has also been proposed. For example, Jimenez \& Haiman (2006) argue that many puzzles related to Lyman Break Galaxies at $z \gtrsim 4$ would be solved by assuming that a fraction of their light is produced by metal-free stars. Similarly, Malhotra \& Rhoads (2002) suggest that the large equivalent width of Ly $\alpha$ emitters at $z>5$ might be a signature of Population III stars (Tumlinson et al. 2003; Scannapieco et al. 2003). However co-existence of both Population III and II stars in the same galaxy appears to require a fine tuning of the star formation history, especially because the initial mass function (IMF) of metal-free stars is probably more biased toward large masses than that of Population II stars. In addition, it would be extremely hard to avoid mixing of of metal-free and metal polluted gas in a galaxy, because of the turbolent nature of the ISM (e.g. see Balsara \& Kim 2005; Balsara et al. 2008). Therefore a significant population of primordial stars in Lyman Break Galaxies is unlikely. Another indirect fingerprint of Population III stars is the presence of peculiar abundance patterns in the gas chemically enriched by Population III stars, with enhanced even-odd element abundance ratios (Heger \& Woosley 2002; Tumlinson et al. 2004), but again no strong evidence is available. Finally, the transition from Population III to Population II star formation leaves an imprint in the reionization history of the Universe (Shull \& Venkatesan 2008; see also Bagla et al. 2009).

The null result achieved so far is not surprising. In fact, the task is very challenging: the expected top-heavy IMF implies that the lifetime of a single Population III star is rather short - possibly only a few million years - and they form in isolation, at least in minihalos (e.g., O’Shea \& Norman 2008). These objects are extremely faint at cosmological distances $\left(m_{\mathrm{AB}}>40\right.$ at $\left.z>10\right)$ and thus unlikely to be detected directly via imaging even with future facilities such as the James Webb Space Telescope (JWST). A better chance 
of detection might be afforded by pair-instability supernovae. In fact, metal-free stars in the mass range $140-260 \mathrm{M}_{\odot}$ end their lives with powerful explosions (up to $10^{53}$ ergs released) powered by the production of $e^{+} e^{-}$pairs (Heger \& Woosley 2002). These are up to 10 times more luminous than standard SNs from Population III stars of lower mass (Heger \& Woosley 2002), although also metal-free stars with $\sim 30-100 \mathrm{M}_{\odot}$ might produce luminous hypernovae with an energy release above $10^{52} \mathrm{ergs}$ (Nomoto et al. 2003). Still, many observational challenges are present at such high redshift $(z \geq 10)$, especially because of the significant time dilation of their light curves (e.g. see Scannapieco et al. 2005; see also Mesinger et al. 2006).

In this paper, we aim to evaluate the number density of pockets of primordial gas that survive to $z<10$, using conservative assumptions on the metal enrichment efficiency and parameterizing the results based on the strength of galactic winds. Even with efficient metal transport, metal-free pockets still survive at $z \approx 6$. Thus if massive stars are formed out of this primordial gas, the expected star formation rate can lead to the detection of hypernovae using all-sky surveys such as the Large Synoptic Survey Telescope (LSST).

The paper is organized as follows. In $\S 2$ we present our model for the formation rate of metal-free halos around the end of the reionization era, whose predictions are discussed in $\S[$ along with the prospects for the observational measures of the metal-free star formation rate after reionization. We conclude in $\S$ 4.

\section{Pristine halo formation rate}

\subsection{Numerical setup}

The numerical simulations have been carried out using the Particle Mesh Tree code Gadget-2 (Springel 2005). We adopt a cosmology based on the fifth year WMAP data (Komatsu et al. 2008): $\Omega_{\Lambda}=0.72, \Omega_{m}=0.28, \Omega_{b}=0.0462, H_{0}=70 \mathrm{~km} \mathrm{~s}^{-1} \mathrm{Mpc}^{-1}$ and $\sigma_{8}=0.817$, where $\Omega_{m}$ is the total matter density and $\Omega_{b}$ is the baryon matter density in units of the critical density, $\rho_{c}=3 H_{0}^{2} /(8 \pi G)$. Here, $H_{0}$ is the Hubble constant parameterized as $H_{0}=100 h \mathrm{~km} \mathrm{~s}^{-1} \mathrm{Mpc}^{-1}, \Omega_{\Lambda}$ is the dark energy density, and $\sigma_{8}$ is the root mean squared mass fluctuation in a sphere of radius $8 h^{-1} \mathrm{Mpc}$ extrapolated to $z=0$ using linear theory. The initial conditions have been generated with a code based on the Grafic algorithm (Bertschinger 2001) using a $\Lambda C D M$ transfer function computed via the fit by Eisenstein \& Hu (1999) with spectral index $n_{s}=0.96$.

We start our principal simulation at redshift $z=199$ using a box of edge $l=7 h^{-1} \mathrm{Mpc}$, $N=1024^{3}$ particles, a mass resolution of $3.4 \times 10^{4} \mathrm{M}_{\odot}$ and a force resolution of $0.16 \mathrm{~h}^{-1} \mathrm{kpc}$. 
A total of 79 snapshots are saved from $z=30$ to $z=4$, and from these snapshots dark matter halos are identified with a friend-of-friend halo finder (Davis et al. 1985) using a linking length equal to 0.2 of the mean particle separation. An additional, lower resolution simulation $\left(N=512^{3}, l=10 h^{-1} \mathrm{Mpc}\right)$ is described in Appendix $\mathrm{A}$, where we discuss the convergence of our results.

For every redshift $z$, we define a minimum halo mass for primordial star formation which is the lowest halo mass that can efficiently cool in a Hubble time. Two main coolants are available for metal-free gas: molecular hydrogen and atomic hydrogen (see Tegmark et al. 1997) with different efficiencies depending on the gas temperature. At the time of formation of the halo the gas temperature is approximately equal to the halo virial temperature,

$$
T_{\text {vir }}(M, z)=2554 \mathrm{~K}\left(\frac{M}{10^{6} \mathrm{M}_{\odot}}\right)^{2 / 3}\left(\frac{1+z}{31}\right) .
$$

Molecular hydrogen is an effective coolant at virial temperatures $T_{\text {vir }} \gtrsim 10^{3} \mathrm{~K}$ Galli \& Palla 1998), but it can be photodissociated by radiation in the $\mathrm{H}_{2}$ Lyman-Werner bands (Lepp \& Shull 1983), which progressively builds up as the redshift decreases and the Universe starts to be ionized. Extended Press-Schechter modeling shows that this cooling channel is significantly quenched at $z \gtrsim 15$ (Haiman et al. 1997; Haiman \& Bryan 2006; Trenti \& Stiavelli 2009), at which point metal-free star formation shifts to halos with $T_{\text {vir }} \gtrsim 10^{4} \mathrm{~K}$, where self-shielding from the Lyman-Werner background is efficient and atomic hydrogen cooling is also possible. We therefore assume that the minimum halo mass capable of cooling is that derived in the reference model of Trenti \& Stiavelli (2009), which includes the effect of a self-consistent Lyman-Werner background. This minimum mass versus redshift, $M_{\min }(z)$, is presented in Fig. 1. Given the mass resolution of our simulation, we can resolve halos capable of forming Population III stars via $\mathrm{H}_{2}$ cooling up to $z \leq 26$ with $N \geq 32$ particles. For $z \leq 13$ the minimum halo mass capable of forming stars corresponds to that of halos with $T_{\text {vir }} \geq 10^{4} \mathrm{~K}$, which can be written as:

$$
M_{\min }(z \leq 13)=\left(7.75 \times 10^{6} \mathrm{M}_{\odot}\right)\left(\frac{1+z}{31}\right)^{-3 / 2} .
$$

As a conservative upper limit to the self-enrichment of halos, we flag all particles of a halo with mass $M>M_{\min }(z)$ as "metal enriched". We further assume that a necessary, but not sufficient condition for a halo to be pristine is to be made entirely of particles that have never been in a halo capable of cooling at higher redshift.

Metal enrichment is also possible due to supernovae winds originating in nearby halos. Wind bubbles during the reionization epoch can propagate out to a comoving radius of 
$\sim 150 h^{-1} \mathrm{kpc}$ by redshift $z=6$ accordingly to the Furlanetto \& Loeb (2003) model (but Madau et al. 2001 predict bubble radii approximately two times smaller). An estimate of the wind bubble radius can be also obtained noting that a bubble expanding at $\sim 60 \mathrm{~km} \mathrm{~s}^{-1}$ (Furlanetto \& Loeb 2003) reaches a radius of $\sim 150 h^{-1} \mathrm{kpc}$ after $500 \mathrm{Myr}$. Metals are in fact assumed to be transported by winds at high speed $\left(\sim 300 \mathrm{~km} \mathrm{~s}^{-1}\right)$ until they mix in a thin spherically symmetric shell, propagating at $\sim 60 \mathrm{~km} \mathrm{~s}^{-1}$, that eventually fragments by cooling and pollutes the IGM (Furlanetto \& Loeb 2003). The simple picture discussed here is consistent with more detailed modeling of the interaction of the winds with the IGM. Using the non-cosmological Sedov-Taylor model, as written in equation 8 of Tumlinson et al. (2004), we obtain bubble sizes of $\lesssim 150 h^{-1} \mathrm{kpc}$ at $z \sim 6$.

To derive a conservative upper limit to the wind enrichment, we compute for every halo that is not self-enriched the distance to neighbor halos that have experienced star formation in the past, that is of mass $M>M_{\min }$. The size of the wind bubble around these halos depends on the first time they experienced star formation, assuming a bubble expansion speed in the range $v_{\text {wind }}=40-100 \mathrm{~km} \mathrm{~s}^{-1}$. If the distance between the non-self-enriched halo and other halos is greater than the wind bubble sizes, we have a truly pristine halo. No metal wind had the opportunity to reach the halo (see also the discussion of metal enrichment in Ricotti et al. 2008).

This assumption is in reality likely to overestimate the efficiency of wind pollution. As the wind bubble expands, its capability of enriching the IGM above the critical metallicity needed to quench Population III formation is progressively reduced. For example, if we assume a critical metallicity of $10^{-3.5} Z_{\odot}$, a non self-enriched halo of mass $10^{8} \mathrm{M}_{\odot}$ needs to be polluted with $\sim 100 \mathrm{M}_{\odot}$ of metals by winds. We assume that the galaxy with the winds has a star formation efficiency $\epsilon=10^{-2}$ and a metal yield of $Y \sim 2.4 \times 10^{-2}$ (total mass of metals released per unit solar mass in the IMF). This value is estimated by assuming a $3 \mathrm{M}_{\odot}$ metal release from a supernova and one supernova formed for every $120 \mathrm{M}_{\odot}$ of mass in the IMF. The wind then contains about $3960 \mathrm{M}_{\odot}$ of metals for a wind galaxy also hosted in a $10^{8} \mathrm{M}_{\odot}$ halo. Therefore a non self-enriched halo needs to have a cross section with the wind bubble of at least $100 / 3960 \times 4 \pi \approx 0.32$ sr in order to be polluted above the critical metallicity assuming a $100 \%$ efficiency in the pollution process. Given that a $10^{8} \mathrm{M}_{\odot}$ halo has a (comoving) virial radius $\sim 10.5 h^{-1} \mathrm{kpc}$ at $z=6$, the maximum radius of the wind bubble is only $\sim 33 h^{-1} \mathrm{kpc}$. Of course, multiple bubbles can contribute to the metal enrichment of a halo, and this enrichment can happen before the virialization process is completed. The gas is then less concentrated and has a larger cross section. 


\subsection{Results}

In Fig. 2 we plot the formation rate $\left(d^{2} n / d t d V\right)$ of halos that are not self-enriched. This quantity is slowly declining from $z=25$ to $z=13$ and exhibits a local maximum at $z \sim 10$, at which time about 30 halos are formed per year per comoving $\mathrm{Gpc}^{3}$. Interestingly, their formation rate is only slowly varying in the redshift range $7 \lesssim z \lesssim 25$ because of two competing effects. First, overdensities that lead to these halos become progressively more efficient, and second, as time passes, the supply of metal-free gas decreases and it is more probable that a region has been self-enriched. The halo formation rate starts dropping rapidly only at $z \lesssim 7$. The evolution of $d^{2} n / d t d V$ measured in our numerical simulation is in excellent agreement with the Trenti \& Stiavelli (2009) model, shown as the red-dashed line in Fig. 2. There is a discrepancy by a factor $\sim 2$ at $z \gtrsim 15$, probably related to the finite resolution of the numerical simulation which does not allow us to resolve the progenitor of halos formed at these redshifts. The agreement improves to $\sim 30 \%$ at lower redshift. With the aid of the analytic model, we can understand the origin of the local maximum in the halo formation rate at $z \sim 10$ (see Fig. 1 in Trenti \& Stiavelli 2009) as associated with the peak of Population III stars formed in halos with $T_{\text {vir }}=10^{4} \mathrm{~K}$. The decline from $z \sim 25$ to $z \sim 13$ is instead associated with Population III formation in minihalos, progressively quenched by the rising Lyman-Werner background.

The spatial distribution of these halos is consistent with that of the general population of halos with the same mass, independent of their enrichment history, as we have verified by measuring the two-point correlation function and thus their bias. This result is natural in the context of extended Press-Schechter modeling, because self-enrichment depends only on density fluctuations at scales smaller than the halo mass.

Dependence on the environment is present instead in the case of wind enrichment. To illustrate the effect of wind enrichment, we plot in Fig. 3 a distance vs. mass scatter plot for the nearest neighbors of non self-enriched halos formed at $z=6$. The halos closest to those that have not been self-enriched are typically small $\left(10^{8} \mathrm{M}_{\odot}\right)$ and at distances comparable to those over which the wind bubbles can propagate at $z=6$. Metal enrichment of non-self enriched halos is thus dominated by dwarf-like galaxies, similarly to what happens in the local universe (Stocke et al. 2006; see also Fujita et al. 2004). About 20\% of the non self-enriched halos have no neighbors within $150 \mathrm{~h}^{-1} \mathrm{kpc}$, a distance that essentially guarantees that these halos contain genuine metal-free gas. Depending on details of the wind propagation, up to $50 \%$ of these halos can be intrinsically metal free. The median of the distance distribution in Fig. 3 is $82.4 h^{-1} \mathrm{kpc}$, for which a single wind bubble is unlikely to chemically enrich a metal-free halo above the critical metallicity for quenching Population III formation. To

quantify the wind enrichment we compute the wind bubble size for all the star-forming halos 
in our simulation as described in $\S 2.1$. We plot the resulting metal-free halo formation rate in Fig. 4 for three different values of the wind bubble expansion velocity, $v_{\text {wind }}=40,60,100$ $\mathrm{km} \mathrm{s}^{-1}$. Even in the "worst case scenario" of propagation at $100 \mathrm{~km} \mathrm{~s}^{-1}$, metal-free halos continue to exist down to $z \sim 5$.

\subsection{Absorption Systems Predictions}

These metal-free halos leave an observational signature as absorption systems before star formation is associated with them. To estimate their column density, we assume that the baryon profile follows the dark matter, a reasonable assumption immediately after virialization and before significant cooling takes place. Assuming a helium mass fraction $Y=0.2477$ and a WMAP-5 cosmology (Komatsu et al. 2008), we can write the typical hydrogen column density of an halo of total mass $M_{h}$ as:

$$
\left\langle N_{H}\right\rangle=10^{20} \mathrm{~cm}^{-2}\left(\frac{\Omega_{m} \mathrm{~h}^{2}}{0.1372}\right)\left(\frac{M_{h}}{10^{8} \mathrm{M}_{\odot}}\right)^{1 / 3}\left(\frac{1+z}{7}\right)^{2} .
$$

If the gas spends 5 Myr before cooling and initiating the protostellar core formation, we

estimate from Fig. 4 that the number density of metal-free absorbers is $\sim 25 \operatorname{arcmin}^{-2}$ at $z=6$. Their physical size is

$$
R_{\mathrm{vir}}=\left[\frac{3 M_{h}}{4 \pi \rho_{\mathrm{vir}}}\right]^{1 / 3} \approx(15.3 \mathrm{kpc})(1+z)^{-1}\left(\frac{M_{h}}{10^{8} \mathrm{M}_{\odot}}\right)^{1 / 3}
$$

and their covering factor on the sky is $\sim 5 \times 10^{-3}$ for $z \in[5.5: 6.5]$. The column density of these system is at the edge of the Damped Ly $\alpha$ (DLA) limit and thus well within the observational limits of both Ly $\alpha$ absorption or 21-cm emission studies. The main limitation for the detection in absorption is the very low number of bright QSOs known at $z>6$ (Fan et al. 2006). In addition, the number density of metal-enriched DLAs is higher by a factor of $\sim 300$ compared to that of metal-free systems if we extrapolate to $z \sim 6$ the intrinsic density of neutral gas found at $z \sim 4.5$ in $\operatorname{DLAs}\left(\Omega_{H I}^{(D L A)}(z=4.5) \sim 10^{-3}\right.$; see Trenti \& Stiavelli 2006) Obtaining strong limits on the metallicity of a $z \sim 6$ absorber will be challenging even for a $30 \mathrm{~m}$ class telescope.

\section{Population III stars and prospects for their detectability}

From the metal-free halo formation rate we can derive a Population III star formation rate once we assume an efficiency for the conversion of gas in stars, a poorly constrained 
parameter. Numerical simulation of the collapse and cooling of metal-free halos seem to favor massive Population III stars formed in isolation, even in halos with virial temperature $T_{\text {vir }} \approx 10^{4} \mathrm{~K}$ in the presence of a strong Lyman-Werner background (see O'Shea \& Norman 2008). However, these simulations are limited to the formation of protostellar cores and are not able to reach the point of actual creation of the star. In addition, cosmological simulations focus on Population III star formation at the start of reionization at $z \gtrsim 15$. The physical conditions at the end of the reionization epoch are different. In particular, the CMB temperature floor is significantly lower, and a strong ionizing UV background is present, which may lead to a change in the initial mass function of Population III stars as suggested by Yoshida et al. (2007). Late time metal-free stars could have masses of 30-50 $M_{\odot}$, rather than the values $M \gtrsim 100 M_{\odot}$ suggested at higher redshift. Note also that, while the very massive star hypothesis is a strong prediction of numerical simulations of Population III star formation, there is some tension with the mass function needed to explain the abundance patterns of extremely metal poor stars in the Milky Way (see Tumlinson 2006). Here we explore these two different scenarios for the formation of metal-free stars at the edge of the reionization, by assuming (i) very massive stars formed in isolation and (ii) clusters of massive stars.

\subsection{Detection during the main sequence lifetime}

First, we evaluate the luminosity of Population III stars on the main sequence. Under the assumption that these stars are massive, we find their main sequence luminosity to be $3 \times 10^{21} \mathrm{erg} \mathrm{s}^{-1} \mathrm{~Hz}^{-1} \mathrm{M}_{\odot}^{-1}$ (Bromm et al. 2001b), which translates into a $1 \mu \mathrm{m}$ observed magnitude $m_{\mathrm{AB}} \approx 40$ at $z=6$ for a $100 M_{\odot}$ star. Therefore, direct detection will be extremely challenging (see Table 1). This is essentially hopeless if star formation happens in isolation. Even if the massive star formation formation occurs in clusters, significant problems are present. For example, a cluster formed with star formation efficiency $\epsilon=10^{-2}$ in a $10^{8} \mathrm{M}_{\odot}$ halo a would have an observed $1 \mu \mathrm{m}$ luminosity $m_{\mathrm{AB}} \approx 32$.

In our model with $v_{\text {wind }}=60 \mathrm{~km} \mathrm{~s}^{-1}$, we expect a number density on the sky of $\sim 10$ $\operatorname{arcmin}^{-2}$ i-dropouts 1 Population III sources at $5.5 \lesssim z \lesssim 6.5$ (assuming that each source remains visible for $2 \mathrm{Myr}$ ). Detecting them requires significantly deeper exposures than the HST-UDF, as those observations only reach down to $z_{850 L P}=28.6$ at $5 \sigma$ (Oesch et al. 2009). Even JWST might not be sufficient, unless the Population III star formation efficiency is

\footnotetext{
${ }^{1}$ These are high-redshift sources identified in broad-band imaging by inferring the location of the Ly $\alpha$ IGM-absorption break, see Steidel et al. (1996).
} 
higher than $\epsilon=10^{-2}$ (see also Johnson et al. 2009). In fact, a deep-field JWST integration is expected to reach $m_{z} \approx 31$ in 65 hours (see Table 1). Adopting a different strategy and searching for Ly $\alpha$ emission via narrow-band filters would require a sensitivity of $3 \times$ $10^{41} \mathrm{erg} \mathrm{s}^{-1}$ for the same sources (Bromm et al. 2001b) with an expected number density $\sim 0.5 \operatorname{arcmin}^{-2}$. The lower number density on the sky is given by the smaller redshift range probed by narrow band filters $(\Delta z \sim 0.05$ versus $\Delta z \sim 1$ in broad-band imaging). Current 8 $\mathrm{m}$ class telescopes reach a sensitivity of $10^{43} \mathrm{erg} \mathrm{s}^{-1}$ at $z \sim 7$, equivalent to an observed flux of $2 \times 10^{-17} \mathrm{erg} \mathrm{s}^{-1} \mathrm{~cm}^{-2}$ (Ota et al. 2008). A $30 \mathrm{~m}$ class telescope is expected to improve this limit by about a factor 50 , reaching $\sim 10^{-19} \mathrm{erg} \mathrm{s}^{-} 1 \mathrm{~cm}^{-2}$, which might be sufficient to detect Ly $\alpha$ emission from a large cluster of Population III stars.

Even if future telescopes reach the sensitivity required to detect Population III clusters, the main problem is how to distinguish them from Population II sources. Adopting the

luminosity function measured at redshift $z=6$ from the UDF survey (Bouwens et al. 2006) and extrapolating it down to $m_{z}=32$, we expect a number density of $\sim 42 \operatorname{arcmin}^{-2}$ idropouts from metal-enriched galaxies. While contamination from Population II stars can in principle be addressed owing to the higher effective temperature of Population III stars (Tumlinson \& Shull 2000), it will be extremely challenging to identify the characteristic He II emission features expected from higher temperatures, for sources near the detection limit of an imaging-only survey. Therefore, disentangling the relative contribution does not appear feasible, especially given the intrinsic uncertainties on the luminosity function at high redshift (see Trenti \& Stiavelli 2008). Although Population III sources at lower redshift $(z \approx 5)$ will appear brighter than their $z \approx 6$ counterparts, their direct identification as V-dropouts will be even more challenging because of the increased number of Population II V-dropout sources combined with a sharp decline of the number of Population III sources at this redshift (see Fig. 4).

\subsection{Supernovae Detection}

The possibility of detecting Population III stars in their Supernova phase are more promising, especially if these stars are very massive and hypernovae and/or pair instability SNe (PISNe) are preferentially produced. Theoretical modeling of PISNe light curves by Scannapieco et al. (2005) predicts a peak luminosity $M_{\mathrm{AB}} \in[-19:-21.5]$, depending on the mass of the progenitor $\left(M \in[140: 260] \mathrm{M}_{\odot}\right)$. This is in reasonably good agreement with the most luminous SN observed to date, SN2006gy (Smith et al. 2007), but the results by Heger \& Woosley (2002) and Scannapieco et al. (2005) are based on one-dimensional, nonrotating simulations. Thus they carry some uncertainty both in the expected mass range 
for PISN explosions as well as in the total energy released. For example, Ekström et al. (2008) find that inclusion of significant amounts of rotation could enhance the explosion energy. Assuming here the observed light curve of SN2006gy as a reference (but note that SN2006gy is not metal free), we expect a peak luminosity $M_{\mathrm{AB}} \sim-21.8$, which translates into a $0.14 \mu \mathrm{m} M_{\mathrm{AB}} \sim-20.5$ assuming a blackbody spectrum with effective temperature $T_{\text {eff }}=15000 \mathrm{~K}$ (Scannapieco et al. 2005). This leads to an observed $1 \mu \mathrm{m} m_{\mathrm{AB}} \approx 26.25$ at $z \sim 6$ (distance modulus of 48.86 and K-correction of $-2.5 \log _{10}(1+z)$ ). To obtain a robust detection of the supernova peak luminosity curve, a survey reaching down to $m_{\mathrm{AB}} \approx 27.0$ might be adequate, although the near-UV spectrum of SN2006gy is not well constrained. Thus the prediction of its observed luminosity is intrinsically uncertain at $\lambda \lesssim 1 \mu m$ when the source is at $z \sim 6$. Because a pair instability supernova light curve remains near its peak for a relatively long time (two to three months) plus cosmological time dilation for the observer rest frame, a search for $z \sim 6$ SNs would require a multi-year time-frame. This is convenient, as it allows stacking multiple images for recurring all-sky surveys such as LSST. Given the current LSST requirements (see Ivezić et al. 2008) a $5 \sigma$ detection at $m_{\mathrm{AB}}=26.25$ in the $\mathrm{z}$ band cannot be reached within the main survey, but is within reach of a deeper "mini-survey" (see Ivezić et al. 2008 Sec. 3.2.2).

Supernovae detection at $z \sim 5$ does appear potentially within the capabilities of the main LSST survey, even though these sources will be rarer than their $z \sim 6$ counterparts. Population III supernovae at $4.5 \lesssim z \lesssim 5.5$ can be detected with LSST in the i-band, where the instrument is more sensitive by 0.7 magnitudes than in the z-band. In addition, the $z \sim 5$ sources will be about 0.3 magnitudes brighter compared to those at $z \sim 6$. A $4 \sigma$ limit of $m_{i}=25.95$ can be achieved by stacking the 23 individual exposures, each with sensitivity of 24.0, that are planned for each year of operations (see Table 1). Combining the i-band data with those in the z-band will further increase the confidence level at which a source is identified. This strategy will push the observations to their detection limit, but if there is a distribution of peak luminosity, LSST may be able to identify at least the brightest explosions. Of course, with the very large-data data set generated by LSST, some false positives are expected. Follow-up of the candidates will screen out random fluctuations in the photometry. Identifying these transients as intrinsically high redshift supernovae will be facilitated by the deeper $\mathrm{r}$ and g-bands observations (24.7 and 25.0 magntiudes, respectively, for a single visit).

If supernovae from massive Population III stars are still forming at $z \sim 4$, as suggested by Fig. 4, these sources will likely be well within LSST capabilities, thanks to a further reduction in the distance modulus and the increased sensitivity in the $\mathrm{r}$ band compared to the i band: These two factors will yield an overall gain of more than 1 mag, and a $5 \sigma$ detection of a SN2006gy-like source at $z \sim 4$ will require stacking of only 5 images in the 
g-band.

Our prediction for the observed pair instability supernova rate is plotted in Fig. 5 (for $v_{\text {wind }}=60 \mathrm{~km} \mathrm{~s}^{-1}$ ) and shows that about one supernova per year per $\sim 400 \mathrm{deg}^{2}$ of sky is expected at $z=6$, with the rate going down to one per $\sim 1250 \operatorname{deg}^{2}$ at $z=5$. The rate at $z \sim 5$ is high enough to yield up to $\sim 100$ supernovae over the LSST lifetime. Extrapolating the rate for g-dropouts $(z \sim 4)$ supernovae, we still expect a few events per year.

Establishing the high-redshift nature of these transients is expected to be relatively simple, as no other transient objects are known to possess such peak luminosities and light curve properties. A possible source of contamination arises from pair instability supernovae formed from the merger of two massive, metal-enriched stars (Portegies Zwart \& van den Heuvel 2007). Detailed estimates for the pair instability supernova rate from this channel are still lacking. Given the rarity of 2006gy-like supernovae in the local universe, the contamination is not expected to be severe, below 1 event $\mathrm{yr}^{-1}$.

The detection of Population III supernovae will be more difficult if the progenitor stars are only moderately massive (typical masses $30 \mathrm{M}_{\odot}$ ). Still up to $6 \times 10^{51}$ ergs can by released by hypernovae with an initial mass of $20-25 \mathrm{M}_{\odot}$ (Nomoto et al. 2008). However, the supernova rate will be higher, potentially tens of supernovae per $\operatorname{deg}^{2}$ assuming multiple stars per halo, and the peak luminosity of hypernovae might be fainter than that of a pair instability explosion (Nomoto et al. 2003). This will put them outside the reach of LSST, and a specific deep survey should be planned to search for them. First, a more detailed prediction for their light curve is needed. The best-case scenario for supernovae detection would be if multiple, very massive Population III stars formed per halo.

\section{Conclusion}

In this paper, we investigated the possibility of a long tail in the epoch of Population III star formation, extending to $z \leq 7$. This epoch has been suggested to extend to redshifts after reionization because of inhomogeneous IGM metal enrichment and a finite transition from Pop III to Pop II modes of star formation (e.g., Scannapieco et al. 2003; Jimenez \& Haiman 2006; Tornatore et al. 2007; Shull \& Venkatesan 2008). Our simulations reach a mass resolution sufficient to identify Population III star formation driven by $\mathrm{H}_{2}$ cooling at $z \leq 26$ in halos with $M \geq 10^{6} \mathrm{M}_{\odot}$. At the same time, they cover a sufficiently large volume $\left(10^{3} \mathrm{Mpc}^{3}\right)$ to be representative of the Universe at $z \gtrsim 5$ for the halo mass scale relevant to Population

III formation (see Bagla \& Ray 2005). Coupling the dark matter only dynamics with conservative assumptions on self-enriched (genetic) and wind enriched scenarios, we show that 
pockets of metal-free stars clearly exist well after the end of reionization, although with a small number density (one new halo $\mathrm{Gpc}^{-3} \mathrm{yr}^{-1}$ formed at $z=6$ ). Our results confirm the Population III star formation trend identified by Tornatore et al. (2007) for $z \lesssim 6$, although their mass resolution was insufficient to evaluate self-enrichment from minihalos, which might explain the different behaviour at $z>6$ (see Appendix A). However, our inferred Population III star formation rate assumes one very massive star per halo and is smaller by a factor $\sim 100$ at $z \sim 6$ compared to that plotted in Fig. 1 of Tornatore et al. (2007). If we were to assume instead that a fraction $\epsilon \sim 10^{-3}$ of the metal-free gas in converted into Population III stars, then our results would be much closer to those of Tornatore et al. (2007) at $z \lesssim 6$.

While the $z \lesssim 10$ Population III star formation rate is several orders of magnitude lower than that of metal-enriched stars, the presence of metal-free gas at $z \lesssim 6$ opens an interesting window for detecting Population III stars. If their expected initial mass function is peaked toward very massive stars $\left(M \approx 100 \mathrm{M}_{\odot}\right)$ that could thus explode as hypernovae or pairinstability supernovae, their expected rate (see Fig. [5) and peak luminosity of $m_{\mathrm{AB}} \lesssim 26$ at $z \lesssim 5$ suggest that future ground-based searches with LSST can probe this mode of star formation. We predict one $z \approx 5$ Population III supernova per year per $1250 \mathrm{deg}^{2}$. We suggest that large-area blind searches will be necessary to find these objects, as the formation sites for Population III stars are distributed almost uniformly on the sky, far $\left(\sim 150 h^{-1} \mathrm{kpc}\right.$ equivalent to $\sim 5$ arcsec) from the nearest (dwarf) galaxy. A prediction of our model for metal-free supernovae is that no host galaxy will be present. Furthermore, the two point correlation function of these supernovae will show that these sources are significantly less biased than the general population of $\sim 10^{8} \mathrm{M}_{\odot}$ dark matter halos at that redshift (see Fig. 3).

All our assumptions about the enrichment of the IGM and the formation rate of metalfree halos are robust. Thus, the presence of pristine gas available for star formation at $z \lesssim 6$ is a very strong prediction of our modeling. However, translating the amount of gas available into an expected supernova rate carries a major uncertainty, namely the Population III initial mass function and the star formation efficiency. These are highly debated topic, in particular the IMF of Population III stars. In fact some tension between theory/simulations and observations is present (Tumlinson et al. 2004; Tumlinson 2006). In addition, Yoshida et al. (2007) found with a 1-D model for cooling of metal free gas in the presence of a strong UV background, that the IMF of Population III stars is shifted toward smaller masses compared to formation at higher redshift. Under such conditions, it is unclear whether fragmentation will lead to multiple Population III formation. Properly addressing the cooling process of these halos is therefore a fundamental step toward evaluating the Population III formation rate at the edge of the reionization epoch. These issues will be examined in a follow-up paper. 
We thank Željko Ivezić for helpful discussions and an anonymous referee for useful suggestions. MT and JMS acknowledge support from the University of Colorado Astrophysical Theory Program through grants from NASA (NNX07AG77G) and NSF (AST07-07474). MS acknowledges support from NASA (NAG5-12458). Numerical simulations were carried out on the Royal Cluster at STScI.

\section{REFERENCES}

Abel, T., Bryan, G. L., \& Norman, M. L. 2002, Science, 295, 93

Bagla, J. S., Kulkarni, G., \& Padmanabhan, T. 2009, ArXiv e-prints 0902.0853

Bagla, J. S. \& Ray, S. 2005, MNRAS, 358, 1076

Balsara, D. S., Bendinelli, A. J., Tilley, D. A., Massari, A. R., \& Howk, J. C. 2008, MNRAS, 386, 642

Balsara, D. S. \& Kim, J. 2005, ApJ, 634, 390

Beers, T. C. \& Christlieb, N. 2005, ARA\&A, 43, 531

Bertschinger, E. 2001, ApJS, 137, 1

Bouwens, R. J., Illingworth, G. D., Blakeslee, J. P., \& Franx, M. 2006, ApJ, 653, 53

Bromm, V., Ferrara, A., Coppi, P. S., \& Larson, R. B. 2001a, MNRAS, 328, 969

Bromm, V., Kudritzki, R. P., \& Loeb, A. 2001b, ApJ, 552, 464

Bromm, V. \& Larson, R. B. 2004, ARA\&A, 42, 79

Cohen, J. G., Christlieb, N., McWilliam, A., Shectman, S., Thompson, I., Melendez, J., Wisotzki, L., \& Reimers, D. 2008, ApJ, 672, 320

Davis, M., Efstathiou, G., Frenk, C. S., \& White, S. D. M. 1985, ApJ, 292, 371

Eisenstein, D. J. \& Hu, W. 1999, ApJ, 511, 5

Ekström, S., Meynet, G., Chiappini, C., Hirschi, R., \& Maeder, A. 2008, A\&A, 489, 685 
Fan, X., Strauss, M. A., Richards, G. T., Hennawi, J. F., Becker, R. H., White, R. L., Diamond-Stanic, A. M., Donley, J. L., Jiang, L., Kim, J. S., Vestergaard, M., Young, J. E., Gunn, J. E., Lupton, R. H., Knapp, G. R., Schneider, D. P., Brandt, W. N., Bahcall, N. A., Barentine, J. C., Brinkmann, J., Brewington, H. J., Fukugita, M., Harvanek, M., Kleinman, S. J., Krzesinski, J., Long, D., Neilsen, Jr., E. H., Nitta, A., Snedden, S. A., \& Voges, W. 2006, AJ, 131, 1203

Frebel, A., Johnson, J. L., \& Bromm, V. 2007, MNRAS, 380, L40

Fujita, A., Mac Low, M.-M., Ferrara, A., \& Meiksin, A. 2004, ApJ, 613, 159

Furlanetto, S. R. \& Loeb, A. 2003, ApJ, 588, 18

-. 2005, ApJ, 634, 1

Galli, D. \& Palla, F. 1998, A\&A, 335, 403

Haiman, Z. \& Bryan, G. L. 2006, ApJ, 650, 7

Haiman, Z., Rees, M. J., \& Loeb, A. 1997, ApJ, 476, 458

Heger, A. \& Woosley, S. E. 2002, ApJ, 567, 532

Ivezić, Z., Tyson, J. A., \& for the LSST Collaboration. 2008, ArXiv e-prints 0805.2366

Jimenez, R. \& Haiman, Z. 2006, Nature, 440, 501

Johnson, J. L., Greif, T. H., Bromm, V., Klessen, R. S., \& Ippolito, J. 2009, ArXiv e-prints 0902.3263

Komatsu, E., Dunkley, J., Nolta, M. R., Bennett, C. L., Gold, B., Hinshaw, G., Jarosik, N., Larson, D., Limon, M., Page, L., Spergel, D. N., Halpern, M., Hill, R. S., Kogut, A., Meyer, S. S., Tucker, G. S., Weiland, J. L., Wollack, E., \& Wright, E. L. 2008, ArXiv e-prints 0803.0547

Lepp, S. \& Shull, J. M. 1983, ApJ, 270, 578

Madau, P., Ferrara, A., \& Rees, M. J. 2001, ApJ, 555, 92

Malhotra, S. \& Rhoads, J. E. 2002, ApJ, 565, L71

Mesinger, A., Johnson, B. D., \& Haiman, Z. 2006, ApJ, 637, 80

Nagao, T., Motohara, K., Maiolino, R., Marconi, A., Taniguchi, Y., Aoki, K., Ajiki, M., \& Shioya, Y. 2005, ApJ, 631, L5 
Nagao, T., Sasaki, S. S., Maiolino, R., Grady, C., Kashikawa, N., Ly, C., Malkan, M. A., Motohara, K., Murayama, T., Schaerer, D., Shioya, Y., \& Taniguchi, Y. 2008, ApJ, 680,100

Naoz, S., Noter, S., \& Barkana, R. 2006, MNRAS, 373, L98

Nomoto, K., Tanaka, M., Kamiya, Y., Tominaga, N., \& Maeda, K. 2008, in IAU Symposium, Vol. 255, IAU Symposium, ed. L. K. Hunt, S. Madden, \& R. Schneider, 182-188

Nomoto, K., Umeda, H., Maeda, K., Ohkubo, T., Deng, J., \& Mazzali, P. 2003, Nuclear Physics A, 718, 277

Oesch, P. A., Carollo, C. M., Stiavelli, M., Trenti, M., Bergeron, L. E., Koekemoer, A. M., Lucas, R. A., Pavlovsky, C. M., Beckwith, S. V. W., Dahlen, T., Ferguson, H. C., Gardner, J. P., Lilly, S. J., Mobasher, B., \& Panagia, N. 2009, ApJ, 690, 1350

Oh, S. P., Haiman, Z., \& Rees, M. J. 2001, ApJ, 553, 73

O’Shea, B. W. \& Norman, M. L. 2007, ApJ, 654, 66

-. 2008, ApJ, 673, 14

Ota, K., Iye, M., Kashikawa, N., Shimasaku, K., Kobayashi, M., Totani, T., Nagashima, M., Morokuma, T., Furusawa, H., Hattori, T., Matsuda, Y., Hashimoto, T., \& Ouchi, M. 2008, ApJ, 677, 12

Portegies Zwart, S. F. \& van den Heuvel, E. P. J. 2007, Nature, 450, 388

Ricotti, M., Gnedin, N. Y., \& Shull, J. M. 2008, ApJ, 685, 21

Santoro, F. \& Shull, J. M. 2006, ApJ, 643, 26

Scannapieco, E., Madau, P., Woosley, S., Heger, A., \& Ferrara, A. 2005, ApJ, 633, 1031

Scannapieco, E., Schneider, R., \& Ferrara, A. 2003, ApJ, 589, 35

Schneider, R., Omukai, K., Inoue, A. K., \& Ferrara, A. 2006, MNRAS, 369, 1437

Shull, J. M. \& Venkatesan, A. 2008, ApJ, 685, 1

Smith, B. D., Turk, M. J., Sigurdsson, S., O’Shea, B. W., \& Norman, M. L. 2009, ApJ, 691, 441

Smith, N., Li, W., Foley, R. J., Wheeler, J. C., Pooley, D., Chornock, R., Filippenko, A. V., Silverman, J. M., Quimby, R., Bloom, J. S., \& Hansen, C. 2007, ApJ, 666, 1116 
Springel, V. 2005, MNRAS, 364, 1105

Steidel, C. C., Giavalisco, M., Pettini, M., Dickinson, M., \& Adelberger, K. L. 1996, ApJ, 462, L17+

Stocke, J. T., Penton, S. V., Danforth, C. W., Shull, J. M., Tumlinson, J., \& McLin, K. M. 2006, ApJ, 641, 217

Tegmark, M., Silk, J., Rees, M. J., Blanchard, A., Abel, T., \& Palla, F. 1997, ApJ, 474, 1

Tornatore, L., Ferrara, A., \& Schneider, R. 2007, MNRAS, 382, 945

Trenti, M. \& Stiavelli, M. 2006, ApJ, 651, 51

—. 2007, ApJ, 667, 38

—. 2008, ApJ, 676, 767

—. 2009, ApJ, 694, 879

Tumlinson, J. 2006, ApJ, 641, 1

Tumlinson, J. \& Shull, J. M. 2000, ApJ, 528, L65

Tumlinson, J., Shull, J. M., \& Venkatesan, A. 2003, ApJ, 584, 608

Tumlinson, J., Venkatesan, A., \& Shull, J. M. 2004, ApJ, 612, 602

Yoshida, N., Omukai, K., \& Hernquist, L. 2007, ApJ, 667, L117 


\section{A. Validation and Convergence}

To correctly evaluate the number density of metal-free halos around the epoch of reionization, it is necessary to ensure that the numerical simulations we used have a sufficient resolution. Strong winds can originate only from relatively massive halos, and are thus relatively easy to identify. However, self-enrichment is more difficult to model because it may arise primarily from minihalo progenitors. Identifying these halos requires a high mass resolution (single particle mass below $\sim 5 \times 10^{4} \mathrm{M}_{\odot}$ ).

A strong hint that our numerical results have reached convergence with respect to selfenrichment comes from the excellent agreement of the Population III halo formation rate with the analytic model for self-enrichment presented in Trenti \& Stiavelli (2009) (see Fig. 2). In addition, we compare here our production run with $N=1024^{3}$ and a box size of $7 h^{-1} \mathrm{Mpc}$ (single particle mass of $3.4 \times 10^{4} \mathrm{M}_{\odot}$ ) to the results of a control run at lower resolution, with $N=512^{3}$ and box size of $10 h^{-1} \mathrm{Mpc}$ (single particle mass of $7.9 \times 10^{5} \mathrm{M}_{\odot}$ ). In Fig. 6 we plot the corresponding halo formation rate for our reference model with a wind speed of $60 \mathrm{~km} \mathrm{~s}^{-1}$. We clearly see that at $z \lesssim 7$ the two simulations yield the same results. The relative difference in $d^{2} n / d t d V$ is below the variance of the halo number counts in a single snapshot. Note that at higher redshift $(z \sim 15)$ the lower resolution run exhibits a peak in Population III formation rate, which is a numerical artifact induced by the inability to follow self-enrichment due to minihalos formed at $z \gtrsim 20$. The resolution of this "low resolution"

control run is equal to the highest resolution run of Tornatore et al. (2007) suggesting that their results might not be reliable at $z \gtrsim 10$. 


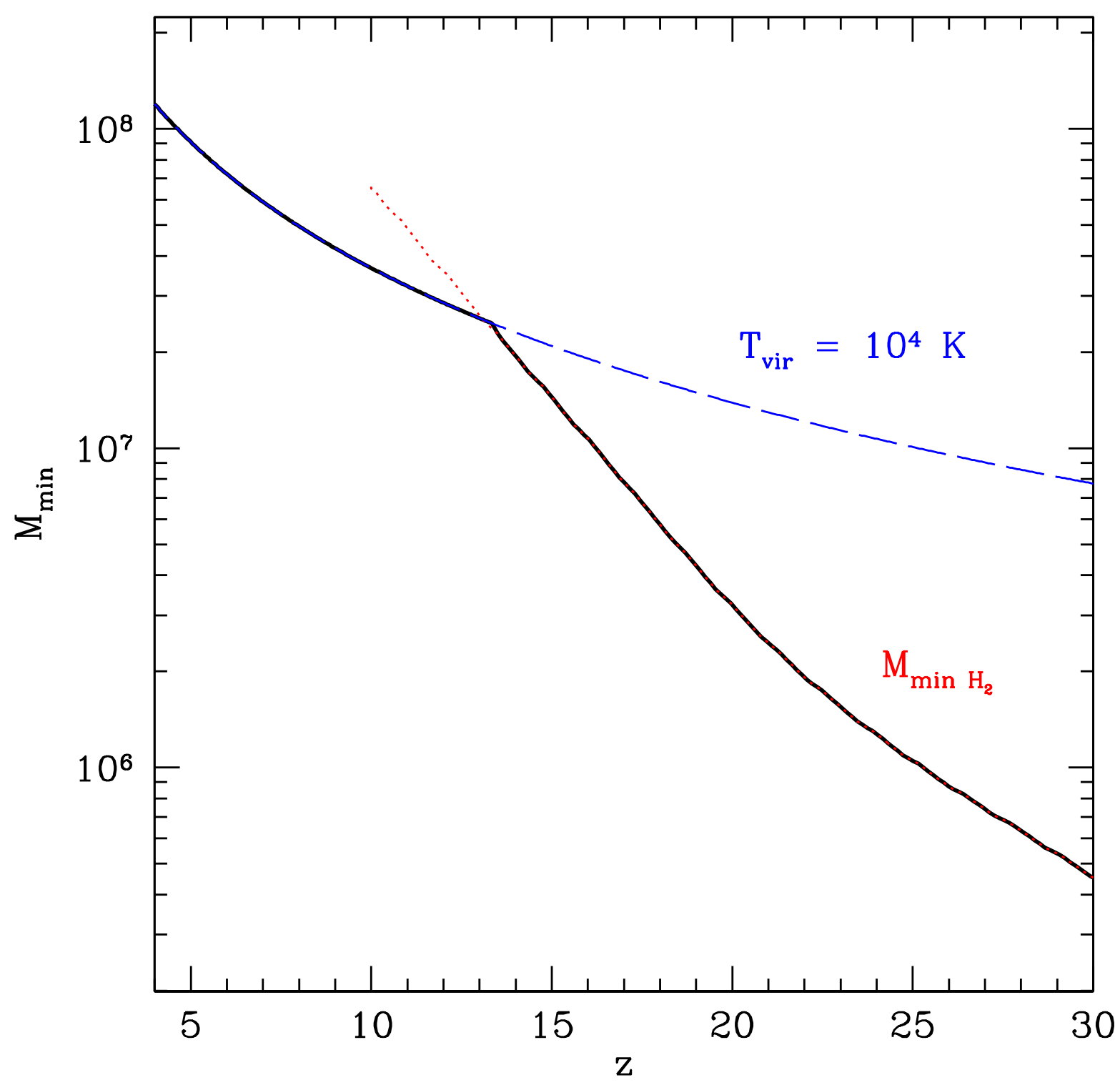

Fig. 1.- Black solid line: minimum halo mass required for cooling and collapse of a metalfree halo in presence of an $\mathrm{H}_{2}$ dissociating Lyman-Werner background. Calculations are based on the standard model by Trenti \& Stiavelli (2009) - see their Fig. 1. For $z<13.4$, molecular hydrogen cooling is strongly suppressed in minihalos, and Population III star formation occurs in halos with virial temperature $T_{\text {vir }}=10^{4} \mathrm{~K}$ (dashed blue line). At higher redshift, cooling is instead possible in minihalos with a minimum mass $M_{m i n H_{2}}$ (red dotted line) self-consistently derived from the Lyman-Werner background created by the first stars and galaxies (see Trenti \& Stiavelli 2009 for details of the model). 


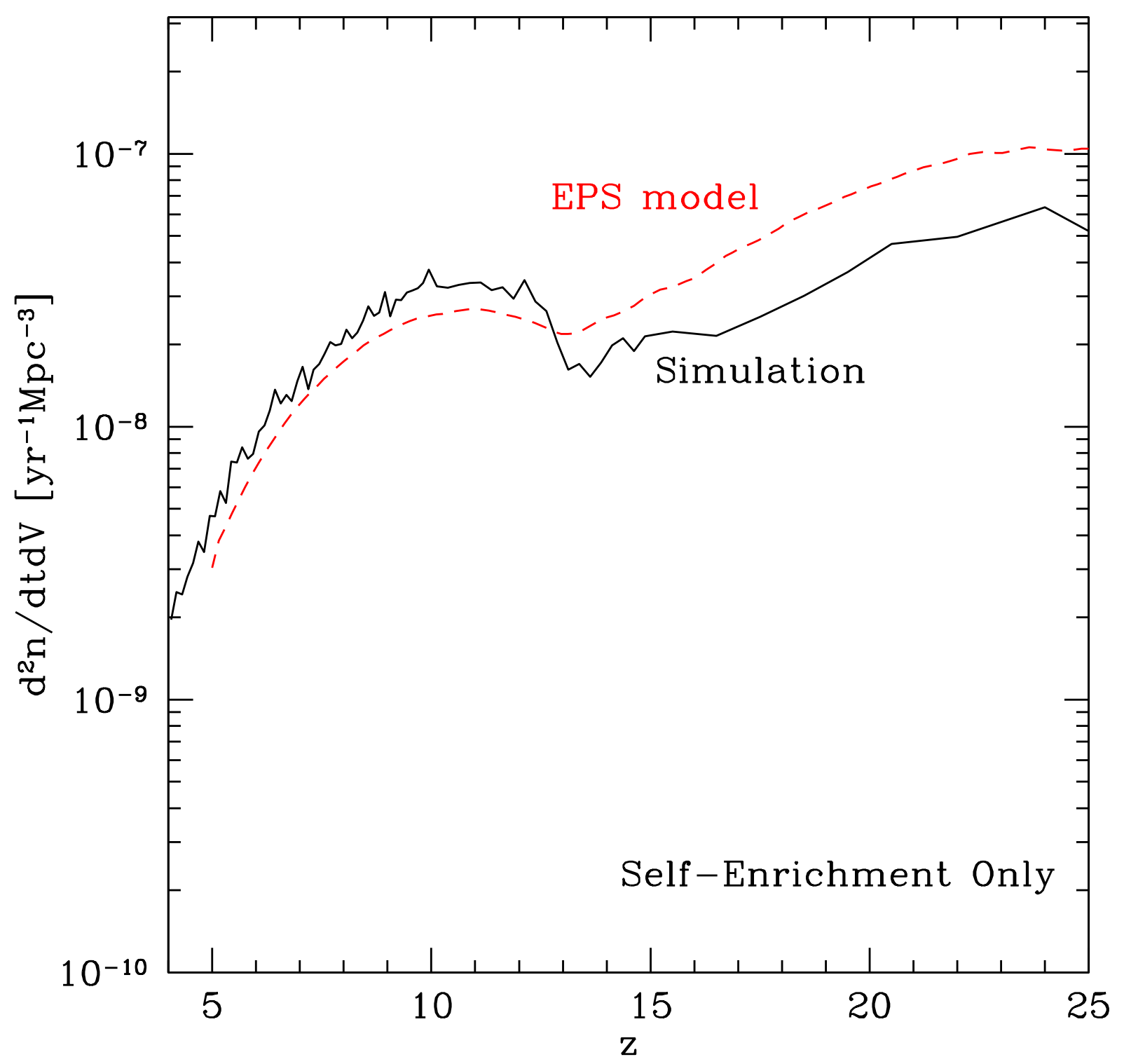

Fig. 2.- Solid black line: formation rate of halos that have not experienced self-enrichment (number of new halos formed per proper unit time (yr) per unit volume (comoving $\mathrm{Mpc}^{3}$ )). The red dashed line is the prediction for the same quantity based on the Extended PressSchechter ("EPS") model of Trenti \& Stiavelli (2009). There is a remarkable agreement especially at redshift $z \lesssim 10$. Winds are not taken into account in this figure. 


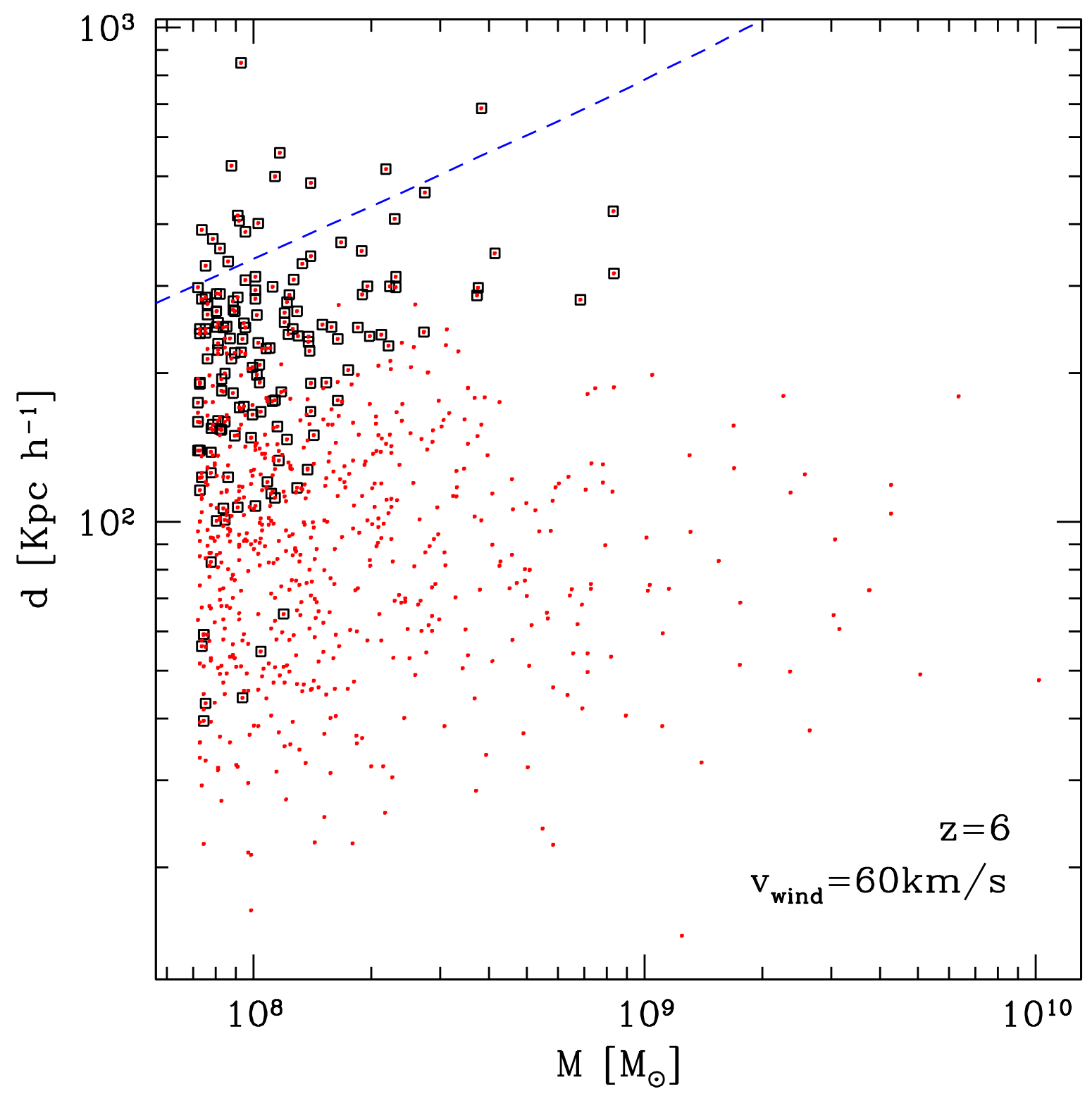

Fig. 3.- Comoving distances and masses of the nearest-neighbor halo of non self-enriched (red dots) and truly pristine halos (black squares) at redshift $z=6$ for a wind speed $v_{\text {wind }}=$ $60 \mathrm{~km} \mathrm{~s}^{-1}$. A significant fraction of halos that were not self-enriched have neighbors too distant to pollute them with their metal winds. The blue dashed line gives the average distance of halos of mass $M$ under the assumption of a uniform distribution, highlighting the effect of clustering. Truly pristine halos are significantly less clustered than their metal enriched counterparts. 


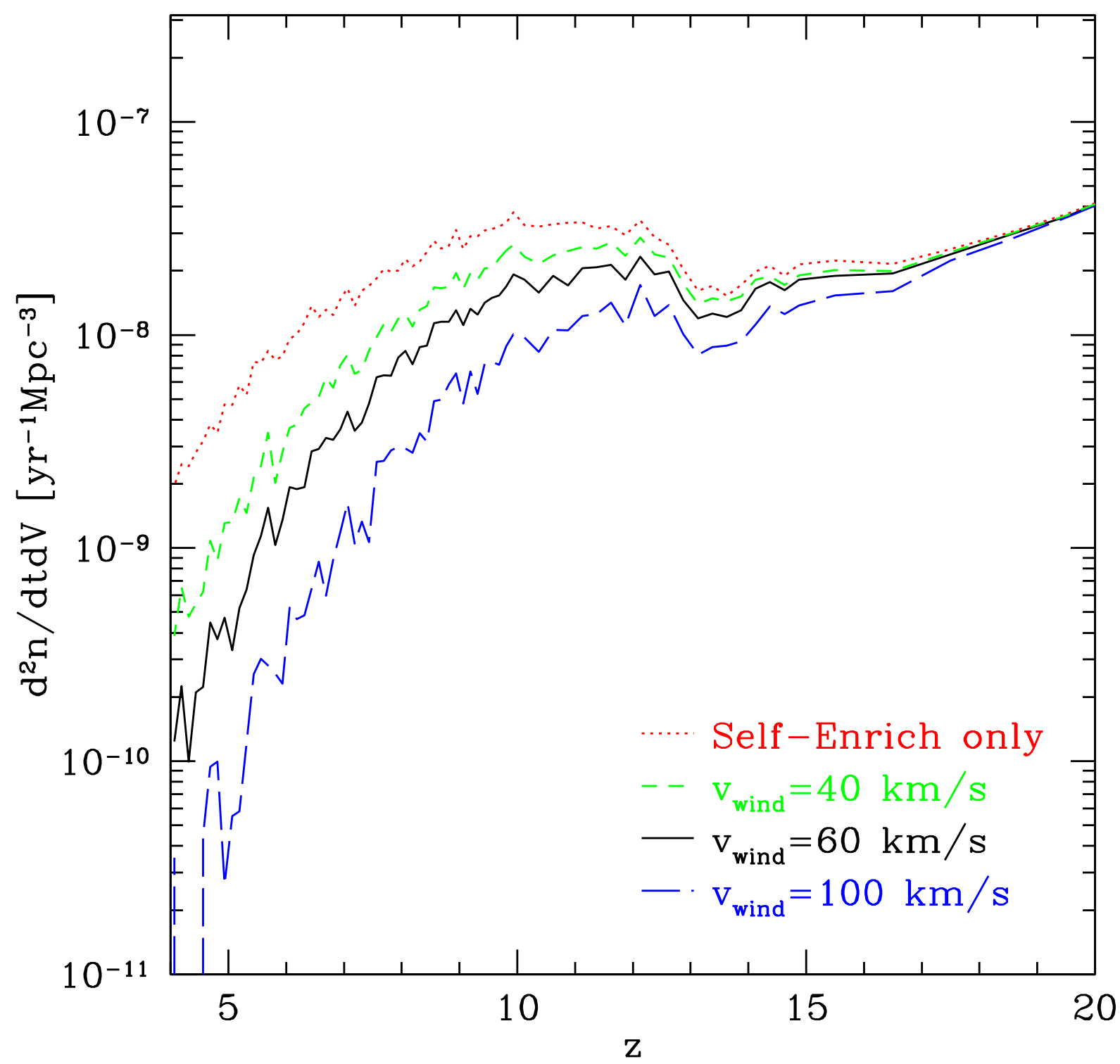

Fig. 4.- Population III halo formation rate after accounting for wind pollution (short dashed green line $v_{\text {wind }}=40 \mathrm{~km} \mathrm{~s}^{-1}$; solid black line $v_{\text {wind }}=60 \mathrm{~km} \mathrm{~s}^{-1}$; long dashed blue line $v_{\text {wind }}=100 \mathrm{~km} \mathrm{~s}^{-1}$ ) compared to the formation rate of halos that were not self-enriched (dotted red line). The red dotted line is also equivalent to assuming $v_{\text {wind }}=0$. 


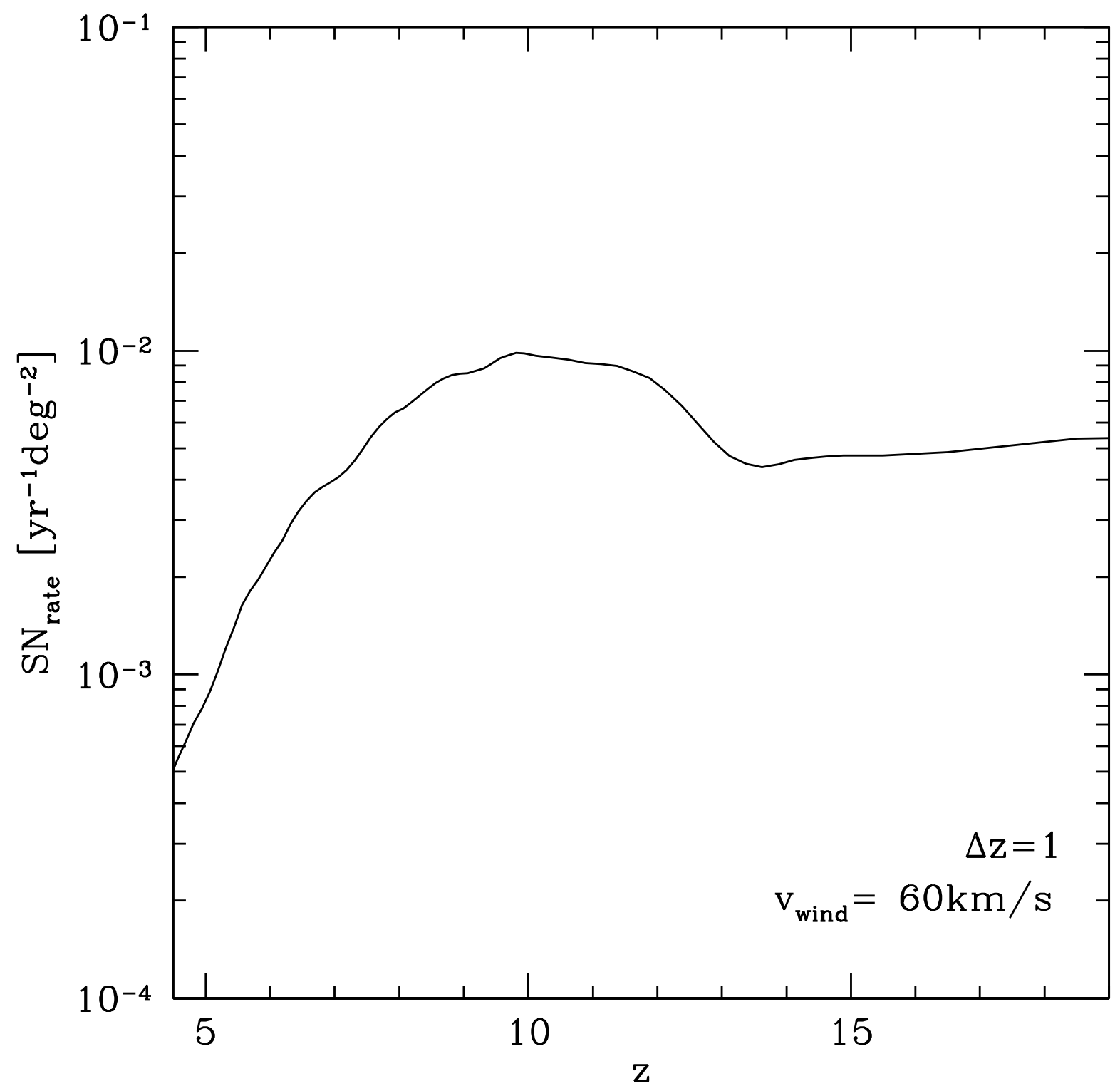

Fig. 5.- Observed Population III supernova rate per year per $\operatorname{deg}^{2}$ as a function of redshift for our standard model of wind propagation $\left(v_{\text {wind }}=60 \mathrm{~km} \mathrm{~s}^{-1}\right)$. This has been obtained assuming one Population III supernova per halo. The field of view depth is $\Delta z=1$ centered at redshift $z$, a choice representative for typical Lyman-Break dropout searches. 


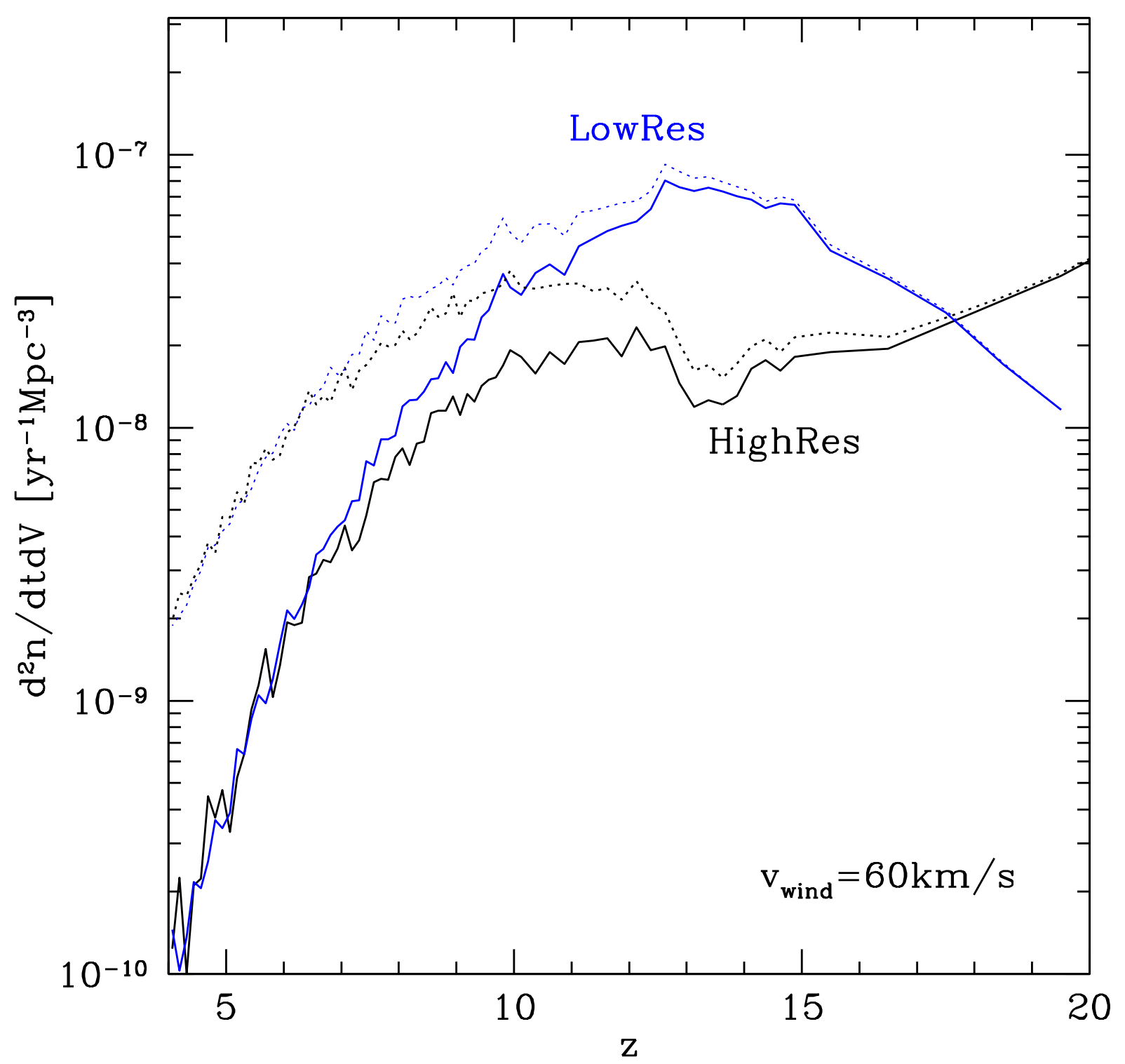

Fig. 6.- Population III halo formation rate obtained with our standard wind prescription $\left(v_{\text {wind }}=60 \mathrm{~km} \mathrm{~s}^{-1}\right)$ from two cosmological simulation, a "Low-Resolution" $N=512^{3}$ run (solid blue line) and a "High-Resolution" $N=1024^{3}$ run (solid black line). The dotted blue and black lines represent the rate in presence of self-enrichment only. The results of the two runs agree well for $z \lesssim 8$. At higher redshift $(8 \lesssim z \lesssim 17$ ), the low resolution run is not able to follow self-enrichment and thus over-estimates the formation rate of metal-free halos. 
Table 1: Sensitivity of current and future facilities for Population III detection

\begin{tabular}{lccccr}
\hline \hline Telescope/Instrument & Band & Sensitivity & Integration Time & Field of view & Time $/\left(10^{3} \mathrm{deg}^{2}\right)$ \\
LSST & $\mathrm{i}$ & $m_{A B}=26$ & $1200 \mathrm{~s}$ & $9.6 \mathrm{deg}^{2}$ & $35 \mathrm{~h}$ \\
SUBARU/Suprime-Cam & $\mathrm{i}$ & $m_{A B}=26$ & $470 \mathrm{~s}$ & $0.25 \mathrm{deg}^{2}$ & $522 \mathrm{~h}$ \\
HST/ACS & F775W & $m_{A B}=26$ & $800 \mathrm{~s}$ & $11.3 \operatorname{arcmin}^{2}$ & $7 \times 10^{4} \mathrm{~h}$ \\
JWST/NIRCAM & F090W & $m_{A B}=26$ & $<30 \mathrm{~s}$ & $9.7 \operatorname{arcmin}^{2}$ & $3093 \mathrm{~h}$ \\
TMT/IRIS $^{a}$ & $\mathrm{i}$ & $m_{A B}=26$ & $\sim 30 \mathrm{~s}$ & $0.06 \operatorname{arcmin}^{2}$ & $5 \times 10^{5} \mathrm{~h}$ \\
\hline JWST/NIRCAM $_{\text {TMT/IRIS }}{ }^{a}$ & F090W & $m_{A B}=31$ & $65 \mathrm{~h}$ & $9.7 \operatorname{arcmin}^{2}$ & $\mathrm{~N} / \mathrm{A}$ \\
TMT & $\mathrm{z}$ & $m_{A B}=31$ & $34 \mathrm{~h}$ & $0.06 \operatorname{arcmin}^{2}$ & $\mathrm{~N} / \mathrm{A}$ \\
\hline
\end{tabular}

Note. - Summary of the sensitivity for $4.5 \lesssim z \lesssim 7$ Population III SNe searches using the current and future facilities listed in the first column. The second column reports the filter used, the third the $5 \sigma$ sensitivity reached in the time given in the fourth column. The fifth column gives the field of view and the last column the time needed to survey $10^{3} \mathrm{deg}^{2}$ : SNe detection is maximally efficient with LSST given its superior field of view. Last two lines: a deep field with JWST NIRCAM or with a $30 \mathrm{~m}$ class telescope will approach the depth necessary for direct imaging of a cluster of Population III stars, if these exist. a: TMT sensitivity is an estimate based on the "TMT Instrumentation and Performance Handbook" (http://www.physics.uci.edu/TMT-Workshop/TMT-Handbook.pdf), as no exposure time calculator is currently available. 